\title{
KRAS rs7973450 A>G increases neuroblastoma risk in Chinese children: a four-center case-control study
}

This article was published in the following Dove Press journal:

OncoTargets and Therapy

Ao Lin, ${ }^{\text {, } * \text { Rui-Xi Hua, }}$,* Jue Tang, '* Jinhong Zhu, ${ }^{3}$ Ruizhong Zhang,' Haixia Zhou, (iD) ${ }^{4}$ Jiao Zhang, ${ }^{5}$ Jiwen Cheng, ${ }^{6}$ Huimin Xia, Jing $\mathrm{He} \mathrm{DI}^{\prime}$

'Department of Pediatric Surgery, Guangzhou Institute of Pediatrics, Guangdong Provincial Key Laboratory of Research in Structural Birth Defect Disease, Guangzhou Women and Children's Medical Center, Guangzhou Medical University, Guangzhou 510623, Guangdong, People's Republic of China; ${ }^{2}$ Department of Oncology, The First Affiliated Hospital of Sun Yat-sen University, Guangzhou 510080, Guangdong, People's Republic of China; ${ }^{3}$ Department of Clinical Laboratory, Biobank, Harbin Medical University Cancer Hospital, Harbin 150040, Heilongjiang, People's Republic of China; ${ }^{4}$ Department of Hematology, The Second Affiliated Hospital and Yuying Children's Hospital of Wenzhou Medical University, Wenzhou 325027, Zhejiang, People's Republic of China; ${ }^{5}$ Department of Pediatric Surgery, The First Affiliated Hospital of Zhengzhou University, Zhengzhou 450052, Henan, People's Republic of China; ${ }^{6}$ Department of Pediatric Surgery, The Second Affiliated Hospital of Xi'an Jiaotong University, Xi'an 710004, Shaanxi, People's Republic of China

*These authors contributed equally to this work

Correspondence: Huimin Xia; Jing $\mathrm{He}$ Department of Pediatric Surgery, Guangzhou Institute of Pediatrics, Guangdong Provincial Key Laboratory of Research in Structural Birth Defect Disease, Guangzhou Women and Children's Medical Center, Guangzhou Medical University, 9 Jinsui Road, Guangzhou 510623, Guangdong, People's Republic of China

Tel +860 2038076001 ;

+860 2038076560

Fax +860 2038076001 ;

+8602038076560

Email xia-huimin@foxmail.com;

hejing198374@gmail.com
Background: Neuroblastoma is one of the most common extracranial solid pediatric tumors. KRAS plays an important role in regulating cell proliferation, differentiation, and apoptosis. Single nucleotide polymorphisms (SNPs) in KRAS have been shown to modify susceptibility to multiple tumors, but no specific molecular epidemiology study was reported regarding neuroblastoma.

Methods: We conducted a four-center case-control study to explore the association between KRAS gene polymorphisms (rs12587 G>T, rs7973450 A>G, rs7312175 G>A) and neuroblastoma susceptibility with 505 Chinese children and 1070 matched controls.

Results: We found that $\mathrm{rs} 7973450 \mathrm{~A}>\mathrm{G}$ was associated with significantly increased neuroblastoma risk [GG vs. AA: adjusted odds ratio $(\mathrm{OR})=4.26,95 \%$ confidence interval (CI) $=1.28-14.19, P=0.018$; GG vs. AA/AG: adjusted $\mathrm{OR}=4.27,95 \% \mathrm{CI}=1.28-14.24, P=0.018]$. The stratified analysis further demonstrated that rs7973450 GG genotype carriers had a higher risk to develop neuroblastoma in the subgroups of males, tumor originated from the adrenal gland and clinical stages III+IV.

Conclusions: Overall, our results suggested that rs7973450 A>G was associated with increased neuroblastoma risk.

Keywords: neuroblastoma, KRAS, polymorphism, susceptibility

\section{Introduction}

Neuroblastoma is one of the most common extracranial pediatric tumors, which accounts for $7-10 \%$ of pediatric tumors worldwide. It has a morbidity of 7.7 per million in China. ${ }^{1,2}$ Neuroblastoma often occurs to children younger than 1-year-old, with an average diagnosis age of 17 months. ${ }^{3}$ Neuroblastoma has diverse clinical phenotypes and its prognosis varies greatly. For instance, a fraction of neuroblastoma patients regress spontaneously. In contrast, about $50 \%$ of patients have high-risk neuroblastoma. ${ }^{4}$ Despite the comprehensive treatment including surgery, chemotherapy, radiotherapy, and autologous stem cell transplantation, the five-year survival rate is still lower than $40 \%$ in high-risk neuroblastoma which accounts for $15 \%$ mortality of early childhood malignant tumor. ${ }^{5}$ Moreover, survivors often sustainably suffer from chronic health problems and have a poor life quality. ${ }^{6}$

The effects of environmental exposures on neuroblastoma are not clear, such as medication, infection and parents' living habits. Significantly statistical result for the association between external factors and neuroblastoma is lacking. ${ }^{7}$ With the development of genome-wide association studies (GWASs), more and more evidence indicated that genetic factors may predispose to neuroblastoma. Genetic differences in individuals mainly result from single nucleotide polymorphisms (SNPs). ${ }^{8} P H O X 2 B^{9}$ and $A L K^{10}$ 
mutations have been shown to contribute to familial neuroblastoma risk. Meanwhile sporadic neuroblastoma susceptibility is related to SNPs in TP53, ${ }^{11,12}$ CASC15, ${ }^{13} M Y C N,{ }^{14}$ BARD1, ${ }^{15,16}$ LMOI $^{17}{ }^{17} X P G,{ }^{18}$ NEFL,${ }^{19}$ CDKN1B, ${ }^{20}$ and HACE1 genes. $^{21}$ Although researchers have extensively explored the genetic etiology of neuroblastoma, many susceptibility genes remain to be determined.

$R A S$ gene family consists of several oncogenes, including $K R A S, N R A S$, and HRAS. KRAS is the most frequently mutated gene in the $R A S$ gene family, mutations in which have been discovered in $1 / 4$ human tumors. K-Ras protein acts as a switch in the RAS-RAF-MEK-MAPK pathway so that regulates cell proliferation, differentiation, and apoptosis by transmitting extracellular signals to the nucleus. $^{22,23} K R A S$ mutations are considered as the first step in tumorigenesis. ${ }^{24}$ Frequent mutations in codon 12 and 13 have been found in a wide spectrum of human tumors, ${ }^{25}$ such as pancreatic cancer ${ }^{24}$ and non-small cell lung cancer. ${ }^{26}$ In recent years, the research focused on $K R A S$ has gradually shifted to the regulation sequence. Polymorphisms in 3' UTRs (rs61764370 T $>\mathrm{G}$, rs712 $\mathrm{T}>\mathrm{G}, \quad$ rs1137282 $\mathrm{A}>\mathrm{G}$ ) and introns (rs12427141 $\mathrm{G}>\mathrm{A}$, rs7315339 $\mathrm{T}>\mathrm{C}$ ) have been observed to significantly modified the susceptibility to lung cancer, ${ }^{27}$ ovarian cancer, ${ }^{28}$ and triple-negative breast cancer. ${ }^{29}$

$K R A S$ mutations have been found in some cases of primary and relapse neuroblastomas. ${ }^{30-32}$ However, there is no orthodox molecular epidemiology study about $K R A S$ and neuroblastoma. Considering the universal importance of the $K R A S$ gene in tumorigenesis, we intended to explore the association between $K R A S$ gene polymorphisms and neuroblastoma susceptibility in Chinese children.

\section{Patients and methods Study population}

We performed a four-center case-control study, which involved 505 patients and 1070 healthy children as described previously (Table S1) ${ }^{33}$ Briefly, patients were confirmed as new neuroblastoma cases by histopathological diagnosis. According to the INSS, patients were divided into clinical stages I, II (IIA, IIB), III, IV, and $4 \mathrm{~S} .{ }^{34,35}$ A total of 1070 healthy children were randomly selected as controls from those who visited these four participating hospitals in the same period. Patients and controls were matching by age, gender, and ethnicity. To achieve relevant legal and ethical requirements, our study was approved by the Institutional Review Committee of four hospitals (the Second Affiliated Hospital and Yuying Children's Hospital of Wenzhou Medical University, the First affiliated Hospital of Zhengzhou University, the Second Affiliated Hospital of Xi'an Jiaotong University, Guangzhou Women and Children's Medical Center). Our study was conducted following the Declaration of Helsinki, and participants or guardians were required to sign informed consent forms. Blood samples were obtained from cases before receiving radiotherapy or chemotherapy.

\section{Genotyping}

We screened potential function polymorphic sites in the $K R A S$ gene by NCBI dbSNP database (http://www.ncbi. nlm.nih.gov/projects/SNP) and SNPinfo (http://snpinfo. niehs.nih.gov/snpfunc.htm). ${ }^{36,37}$ KRAS $\quad$ rs12587 and rs7973450 were predicted to be located in the microRNA binding sites, while rs7312175 in a potential transcription factor binding site. As shown in Figure S1, there exists weak linkage disequilibrium $\left(\mathrm{R}^{2}<0.8\right)$ among rs12587, rs7973450 and rs7312175. The $\mathrm{R}^{2}=0.349$ between rs12587 and rs7973450; $\mathrm{R}^{2}=0.447$ between rs12587 and rs7312175; and $\mathrm{R}^{2}=0.015$ between rs7973450 and rs7312175. TIANamp Blood DNA Kit (TianGen Biotech Co., Ltd., Beijing, China) was used to extract genomic DNA and TaqMan SNP Genotyping Assay (Applied Biosystems, Foster City, CA, USA) for genotyping. ${ }^{38-40}$ To ensure the accuracy, reliability, and repeatability, our study was carried out in strict accordance with the instructions and no falsepositive result was found in the negative control. Besides, $10 \%$ of samples were randomly selected for repeated experiments and the repeatable rate was $100 \%$.

\section{Statistical analysis}

SAS release 9.1 (SAS Institute, Cary, NC, USA) was used for data analysis. Hardy-Weinberg equilibrium (HWE) in controls was estimated by a good-of-fit test. The differences in demographic characteristics and genotype distribution between cases and controls were detected by $t$-test and chi-square test, respectively. For adjusting age and gender, an unconditional multiple logistic regression model was taken to reveal the association between three polymorphisms and neuroblastoma susceptibility. Odds ratios (ORs) and 95\% confidence intervals (CIs) were used to be statistical indicators. Stratified analysis was performed by age, gender, tumor origin and clinical stages. All analyses were two-sided. $P<0.05$ was considered statistically significant. 


\section{Results}

\section{KRAS gene polymorphisms and neuroblastoma susceptibility}

As revealed in Table 1, all of the three polymorphisms in controls conformed to the HWE $(P>0.05)$. Moreover, we found that rs7973450 A $>\mathrm{G}$ was significantly associated with increased neuroblastoma risk (GG vs. AA: adjusted $\mathrm{OR}=4.26,95 \% \mathrm{CI}=1.28-14.19, P=0.018$; and GG vs. AA/ AG: adjusted $\mathrm{OR}=4.27,95 \% \mathrm{CI}=1.28-14.24, P=0.018$ ) after adjusting for age and gender. Unfortunately, we failed to find a significant association with rs $12587 \mathrm{G}>\mathrm{T}$ and rs7312175 G>A.

\section{Stratification analysis}

Compared with AA/AG genotypes carriers, the stratified analysis (Table 2) further revealed that rs 7973450 GG genotype carriers had a higher risk to develop neuroblastoma in the strata of males (adjusted $\mathrm{OR}=10.75,95 \% \mathrm{CI}=1.25$ 92.61, $P=0.031$ ), tumor originated from adrenal gland (adjusted $\mathrm{OR}=6.16,95 \% \mathrm{CI}=1.52-24.94, P=0.011$ ), and clinical stages III+IV (adjusted OR=4.19, 95\% CI=1.0317.02, $P=0.045)$.

\section{Discussion}

KRAS is located in chromosome 12 , coding a KRAS protein with GTPase activity. KRAS protein is activated by attaching to GTP and turned off right after converting the GTP to GDP. As a result, it transmits extracellular signals into the nucleus and regulates the cellular lifecycle of cells. According to previous reports, KRAS not only took part in the RAS-RAF-MEK-MAPK pathway, ${ }^{41}$ but also unit PI3K to jointly activate mTOR. It indicated that KRAS is a key regulatory molecule to cellular growth and proliferation. ${ }^{42}$ What's more, KRAS was associated with

Table I Association between KRAS gene polymorphisms and neuroblastoma risk

\begin{tabular}{|c|c|c|c|c|c|c|c|}
\hline Genotype & Cases $(\mathrm{N}=505)$ & Controls $(\mathrm{N}=1070)$ & $P^{\mathbf{a}}$ & Crude OR $(95 \% \mathrm{Cl})$ & $P$ & Adjusted OR $(95 \% \mathrm{Cl})^{\mathrm{b}}$ & $P^{\mathbf{b}}$ \\
\hline \multicolumn{8}{|c|}{ rs|2587 G>T (HWE=0.287) } \\
\hline GG & $330(65.35)$ & $688(64.30)$ & & 1.00 & & 1.00 & \\
\hline GT & I 46 (28.9|) & $333(31.12)$ & & $0.95(0.77-1.18)$ & 0.653 & $0.94(0.76-1.17)$ & 0.591 \\
\hline TT & $29(5.74)$ & $49(4.58)$ & & $1.29(0.80-2.05)$ & 0.295 & $1.27(0.79-2.02)$ & 0.326 \\
\hline Additive & & & 0.971 & $1.00(0.84-1.20)$ & 0.971 & $1.00(0.83-1.20)$ & 0.998 \\
\hline Dominant & $175(34.65)$ & $382(35.70)$ & 0.685 & $0.96(0.77-1.19)$ & 0.686 & $0.95(0.76-1.19)$ & 0.661 \\
\hline Recessive & $476(94.26)$ & $1021(95.42)$ & 0.321 & $1.27(0.79-2.04)$ & 0.322 & $1.26(0.79-2.03)$ & 0.331 \\
\hline \multicolumn{8}{|c|}{ rs7973450 A>G (HWE=0.080) } \\
\hline AA & $422(83.56)$ & 881 (82.34) & & 1.00 & & 1.00 & \\
\hline AG & $75(14.85)$ & $185(17.29)$ & & $0.88(0.66-1.16)$ & 0.359 & $0.87(0.66-1.16)$ & 0.336 \\
\hline GG & $8(1.58)$ & $4(0.37)$ & & $4.32(1.30-14.40)$ & 0.017 & $4.26(1.28-14.19)$ & 0.018 \\
\hline Additive & & & 0.994 & $1.00(0.77-1.30)$ & 0.994 & $1.00(0.77-1.30)$ & 0.983 \\
\hline Dominant & $83(16.44)$ & $189(17.66)$ & 0.547 & $0.92(0.69-1.22)$ & 0.547 & $0.92(0.69-1.22)$ & 0.540 \\
\hline Recessive & $497(98.42)$ & $1066(99.63)$ & 0.010 & $4.29(1.29-14.31)$ & 0.018 & $4.27(1.28-14.24)$ & 0.018 \\
\hline \multicolumn{8}{|c|}{ rs73।2I75 G>A (HWE=0.130) } \\
\hline GG & $395(78.22)$ & 851 (79.53) & & 1.00 & & 1.00 & \\
\hline GA & $102(20.20)$ & 201 (I8.79) & & I.I2 (0.87-I.44) & 0.393 & I.II (0.86-I.43) & 0.431 \\
\hline AA & $8(1.58)$ & $18(1.68)$ & & $0.98(0.42-2.26)$ & 0.960 & $0.96(0.4 I-2.22)$ & 0.921 \\
\hline Additive & & & 0.621 & $1.06(0.84-1.33)$ & 0.621 & $1.06(0.84-1.33)$ & 0.639 \\
\hline Dominant & $110(21.78)$ & $219(20.47)$ & 0.549 & $1.08(0.84-1.40)$ & 0.549 & $1.08(0.83-1.40)$ & 0.564 \\
\hline Recessive & 497 (98.42) & 1052 (98.32) & 0.887 & $0.94(0.4 I-2.18)$ & 0.888 & $0.93(0.40-2.16)$ & 0.871 \\
\hline \multicolumn{8}{|c|}{ Combined effect of risk genotypes ${ }^{c}$} \\
\hline 0 & $385(76.24)$ & $841(78.60)$ & & 1.00 & & 1.00 & \\
\hline $\mathrm{I}-2$ & $120(23.76)$ & $229(21.40)$ & 0.293 & I.I5 (0.89-I.47) & 0.293 & I. $14(0.89-1.47)$ & 0.303 \\
\hline
\end{tabular}

Notes: The results were in bold, if the $95 \% \mathrm{Cl}$ excluded I or P-values less than $0.05 .{ }^{\mathrm{a}} \chi^{2}$ test for genotype distributions between neuroblastoma patients and cancer-free controls. ${ }^{b}$ Adjusted for age and gender. 'Risk genotypes were rs $12587 \mathrm{TT}$, rs7973450 GG and rs73।2175 GA/AA.

Abbreviations: OR, odds ratio; $\mathrm{Cl}$, confidence interval; $\mathrm{HWE}$, Hardy-Weinberg equilibrium. 


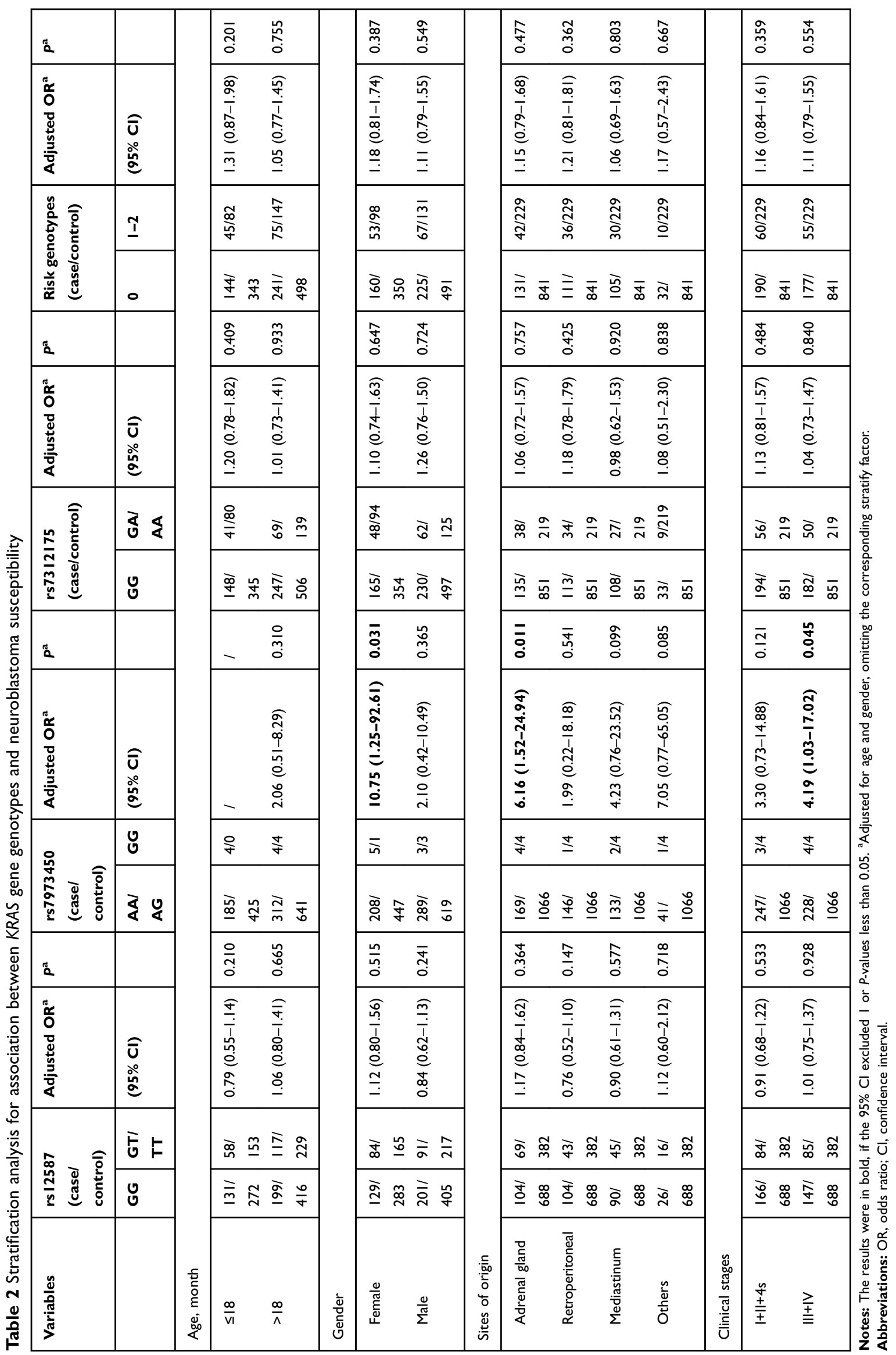


persistent inflammatory responses. ${ }^{43}$ Point mutations in $K A R S$ coding regions (such as codon 12,13) constitutively activated K-Ras protein by increasing GDP/GTP exchange rate or decreasing the GTPase activity. ${ }^{44}$ It continuously activating the relevant pathways and providing a favorable tumor microenvironment for tumor cell growth, survival, invasion, and spread. Instead of altering amino acids or structures, SNPs in $K R A S$ regulatory sequences tended to change $K R A S$ expression. Most of them were highly conserved in evolution. This portended that they would play an indispensable regulating effect on different gene expression processes. ${ }^{45,46}$ A study involving 77 samples have reported that $N A R S$ point mutations were found in 3 samples and no point mutations were found in $H R A S$ and $K R A S{ }^{32}$ Recently, KRAS pGly13Asp and missense mutation were observed in some cases of primary and relapse neuroblastomas. ${ }^{30,31}$

In this study, we draw a conclusion that rs7973450 A>G significantly increased neuroblastoma risk. Unfortunately, we failed to find rs $12587 \mathrm{G}>\mathrm{T}$ and $\mathrm{rs} 7312175 \mathrm{G}>\mathrm{A}$ modified neuroblastoma susceptibility. Interestingly, in our previous study, rs12587 $\mathrm{G}>\mathrm{T}$ was associated with increased nephroblastoma risk, but rs7973450 $\mathrm{A}>\mathrm{G}$ and rs7312175 $\mathrm{G}>\mathrm{A}$ did not modify nephroblastoma susceptibility. ${ }^{36}$ Beside, Dai et al did not observe any association between rs12587 G>T and colorectal cancer, ${ }^{47}$ but Wang et al presented that rs $7312175 \mathrm{G}>\mathrm{A}$ was associated with the recurrence and local dose combination therapy of endometrial cancer. $^{48}$

The rs7973450 was predicted to serve as an important microRNA binding site. Its polymorphism would induce neuroblastoma by abnormally regulating the expression of KRAS and related microRNA. ${ }^{49}$ Moreover, whether the rs 12587 and rs7312175 polymorphisms would be the risk factors for neuroblastoma need further validate.

Take Let-7 complementary sites 6 (LCS6) for example. Chin et al found that $K R A S$ rs61764370 T $>\mathrm{G}$ increased nonsmall cell lung cancer risk. ${ }^{27}$ Further, a double luciferase reporting experiment revealed that rs61764370 $\mathrm{T}>\mathrm{G}$ weakened the inhibitory effect of KRAS 3' UTR by destroying its affinity with Let-7 microRNAs, which resulted in increased K-Ras portent and decreased Let-7 microRNAs. Smits et al indicated that the LCS6 variant seemed to be a primary protective factor for early-stage colorectal cancer susceptibility and prognosis, but it did not associate with advanced colorectal cancer. ${ }^{50}$ In metastatic colorectal cancer, although American patients treated with cetuximab had a better response rate and prognosis when carried $\mathrm{G}$ allele, ${ }^{51}$ an opposite result was observed in the Italians. ${ }^{52}$ In recently, $\mathrm{G}$ allele carriers were shown to increase the risk of chronic myeloid leukemia ${ }^{53}$ and triple-negative breast cancer. ${ }^{29}$ However, whether the LCS6 polymorphism was related to ovarian cancer was uncertain. ${ }^{28,54}$

Based on the appeal examples, there are several reasons why rs7973450 $\mathrm{A}>\mathrm{G}$ and $\mathrm{rs} 7312175 \mathrm{G}>\mathrm{A}$ could not significantly affect neuroblastoma susceptibility in this research. First, the same polymorphism may play different roles in different tumor types, ethnicities and clinical characteristics. Second, one SNP alone was not strong enough to cause tumor, the combination of multiple SNPs might play a significant role in carcinogenesis. ${ }^{55}$ Tonini et al summarized the neuroblastoma susceptibility alleles reported lately, and found that most of neuroblastoma-associated SNPs are located in the genes that were involved in maintaining the chromatin and mitosis integrity. They speculated that the cumulative effect of SNPs could lead to chromosome instability and structural damages during the early embryonic life. ${ }^{56}$ Moreover, environmental exposure and parents' poor living habits might be confusing factors in the association analyze. Finally, the varying incidence rate of the same polymorphism locus in different populations would change the requirement of sample size to detect the real associattion. $^{27}$ It suggests that we should expand sample size, combine other SNPs and control confounders to explore the association between $K R A S$ polymorphisms and tumors in multiple ethnicities. As a case-control study basing hospital, this study inevitably had hospitalization bias.

\section{Conclusion}

In conclusion, with a four-center case-control study in Chinese children, we found the association between neuroblastoma susceptibility and $K R A S$ polymorphisms. Our study suggested that $K R A S$ rs7973450 A $>\mathrm{G}$ significantly increased neuroblastoma risk. The results needed further investigation.

\section{Acknowledgments}

This study was funded by grants from the Pearl River S\&T Nova Program of Guangzhou (No: 201710010086), and Guangdong Provincial Key Laboratory of Research in Structural Birth Defect Disease (No: 2019B030301004).

\section{Disclosure}

The authors report no conflicts of interest in this work. 


\section{References}

1. Matthay KK, Maris JM, Schleiermacher G, et al. Neuroblastoma. Nat Rev Dis Primers. 2016;2:16078. doi:10.1038/nrdp.2016.78

2. Bao PP, Li K, Wu CX, et al. [Recent incidences and trends of childhood malignant solid tumors in Shanghai, 2002-2010]. Zhonghua Er Ke Za Zhi. 2013;51(4):288-294.

3. London WB, Castleberry RP, Matthay KK, et al. Evidence for an age cutoff greater than 365 days for neuroblastoma risk group stratification in the Children's Oncology Group. J Clin Oncol. 2005;23 (27):6459-6465. doi:10.1200/JCO.2005.05.571

4. Cheung NK, Dyer MA. Neuroblastoma: developmental biology, cancer genomics and immunotherapy. Nat Rev Cancer. 2013;13(6):397411. doi: $10.1038 / \operatorname{nrc} 3526$

5. Maris JM, Hogarty MD, Bagatell R, Cohn SL. Neuroblastoma. Lancet. 2007;369(9579):2106-2120. doi:10.1016/S0140-6736(07)60 983-0

6. Laverdiere C, Liu Q, Yasui Y, et al. Long-term outcomes in survivors of neuroblastoma: a report from the Childhood Cancer Survivor Study. J Natl Cancer Inst. 2009;101(16):1131-1140. doi:10.1093/ jnci/djp230

7. Muller-Schulte E, Kurlemann G, Harder A. Tobacco, alcohol and illicit drugs during pregnancy and risk of neuroblastoma: systematic review. Arch Dis Child Fetal Neonatal Ed. 2018;103(5):F467-F473. doi:10.1136/archdischild-2017-313615

8. International Human Genome Sequencing C. Finishing the euchromatic sequence of the human genome. Nature. 2004;431(7011):931945. doi: $10.1038 /$ nature 03001

9. Mosse YP, Laudenslager M, Khazi D, et al. Germline PHOX2B mutation in hereditary neuroblastoma. Am J Hum Genet. 2004;75 (4):727-730. doi:10.1086/424530

10. Mosse YP, Laudenslager M, Longo L, et al. Identification of ALK as a major familial neuroblastoma predisposition gene. Nature. 2008;455 (7215):930-935. doi:10.1038/nature07261

11. Zhang J, Yang Y, Li W, et al. TP53 gene rs1042522 allele G decreases neuroblastoma risk: a two-centre case-control study. $J$ Cancer. 2019;10(2):467-471. doi:10.7150/jca.27482

12. Diskin SJ, Capasso M, Diamond M, et al. Rare variants in TP53 and susceptibility to neuroblastoma. J Natl Cancer Inst. 2014;106(4): dju047. doi:10.1093/jnci/dju061

13. Russell MR, Penikis A, Oldridge DA, et al. CASC15-S is a tumor suppressor lncRNA at the 6p22 neuroblastoma susceptibility locus. Cancer Res. 2015;75(15):3155-3166. doi:10.1158/0008-5472.CAN14-3613

14. Zhou H, Zhuo Z, Chen S, et al. Polymorphisms in MYCN gene and neuroblastoma risk in Chinese children: a 3-center case-control study. Cancer Manag Res. 2018;10:1807-1816. doi:10.2147/CMAR.S1685 15

15. Capasso M, Devoto M, Hou C, et al. Common variations in BARD1 influence susceptibility to high-risk neuroblastoma. Nat Genet. 2009;41(6):718-723. doi:10.1038/ng.374

16. Cimmino F, Avitabile M, Diskin SJ, et al. Fine mapping of $2 \mathrm{q} 35$ high-risk neuroblastoma locus reveals independent functional risk variants and suggests full-length BARD1 as tumor-suppressor. Int $J$ Cancer. 2018;143(11):2828-2837. doi:10.1002/ijc.31822

17. Wang K, Diskin SJ, Zhang H, et al. Integrative genomics identifies LMO1 as a neuroblastoma oncogene. Nature. 2011;469(7329):216220. doi:10.1038/nature09609

18. He J, Wang F, Zhu J, et al. Association of potentially functional variants in the XPG gene with neuroblastoma risk in a Chinese population. J Cell Mol Med. 2016;20(8):1481-1490. doi:10.1111/ jcmm. 12836

19. Capasso M, Diskin S, Cimmino F, et al. Common genetic variants in NEFL influence gene expression and neuroblastoma risk. Cancer Res. 2014;74(23):6913-6924. doi:10.1158/0008-5472.CAN-14-0431
20. Capasso M, McDaniel LD, Cimmino F, et al. The functional variant rs34330 of CDKN1B is associated with risk of neuroblastoma. $J$ Cell Mol Med. 2017;21(12):3224-3230. doi:10.1111/jcmm.13226

21. Diskin SJ, Capasso M, Schnepp RW, et al. Common variation at $6 \mathrm{q} 16$ within HACE1 and LIN28B influences susceptibility to neuroblastoma. Nat Genet. 2012;44(10):1126-1130. doi:10.1038/ng.2387

22. Malumbres M, Barbacid M. RAS oncogenes: the first 30 years. Nat Rev Cancer. 2003;3(6):459-465. doi:10.1038/nrc1097

23. Downward J. Signal transduction. New exchange, new target. Nature. 1998;396(6710):416-417. doi:10.1038/24743

24. Mu DQ, Peng YS, Xu QJ. Values of mutations of K-ras oncogene at codon 12 in detection of pancreatic cancer: 15-year experience. World J Gastroenterol. 2004;10(4):471-475. doi:10.3748/wjg.v10.i4.471

25. Bos JL. ras oncogenes in human cancer: a review. Cancer Res. 1989;49(17):4682-4689.

26. Maki-Nevala S, Sarhadi VK, Ronty M, et al. Hot spot mutations in Finnish non-small cell lung cancers. Lung Cancer. 2016;99:102-110. doi:10.1016/j.lungcan.2016.06.024

27. Chin LJ, Ratner E, Leng S, et al. A SNP in a let-7 microRNA complementary site in the KRAS $3^{\prime}$ untranslated region increases non-small cell lung cancer risk. Cancer Res. 2008;68(20):85358540. doi:10.1158/0008-5472.CAN-08-2129

28. Ratner E, Lu L, Boeke M, et al. A KRAS-variant in ovarian cancer acts as a genetic marker of cancer risk. Cancer Res. 2010;70 (16):6509-6515. doi:10.1158/0008-5472.CAN-10-0689

29. Paranjape T, Heneghan H, Lindner R, et al. A 3'-untranslated region KRAS variant and triple-negative breast cancer: a case-control and genetic analysis. Lancet Oncol. 2011;12(4):377-386. doi:10.1016/ S1470-2045(11)70044-4

30. Pugh TJ, Morozova O, Attiyeh EF, et al. The genetic landscape of high-risk neuroblastoma. Nat Genet. 2013;45(3):279-284. doi:10.10 $38 /$ ng. 2529

31. Schramm A, Koster J, Assenov Y, et al. Mutational dynamics between primary and relapse neuroblastomas. Nat Genet. 2015;47 (8):872-877. doi:10.1038/ng.3349

32. Iolascon A, Badiali M, Pession A, et al. Rare frequencey of point mutations for codon 12,13 and 61 of ras gene in italian neuroblastoma. Int J Oncol. 1993;3(3):529-533. doi:10.3892/ijo.3.3.529

33. Cheng J, Zhuo Z, Xin Y, et al. Relevance of XPD polymorphisms to neuroblastoma risk in Chinese children: a four-center case-control study. Aging (Albany NY). 2018;10(8):1989-2000. doi:10.18632/ aging.101522

34. Luksch R, Castellani MR, Collini P, et al. Neuroblastoma (Peripheral neuroblastic tumours). Crit Rev Oncol Hematol. 2016;107:163-181. doi:10.1016/j.critrevonc.2016.10.001

35. Brodeur GM, Pritchard J, Berthold F, et al. Revisions of the international criteria for neuroblastoma diagnosis, staging, and response to treatment. J Clin Oncol. 1993;11(8):1466-1477. doi:10.1200/JCO.1993.11.8.1466

36. Fu W, Zhuo Z, Hua RX, et al. Association of KRAS and NRAS gene polymorphisms with Wilms tumor risk: a four-center case-control study. Aging (Albany NY). 2019;11(5):1551-1563. doi:10.18632/ aging. 101855

37. Yang T, Wen Y, Li J, et al. NRAS and KRAS polymorphisms are not associated with hepatoblastoma susceptibility in Chinese children. Exp Hematol Oncol. 2019;8:11. doi:10.1186/s40164-019-0135-z

38. He J, Qiu LX, Wang MY, et al. Polymorphisms in the XPG gene and risk of gastric cancer in Chinese populations. Hum Genet. 2012;131 (7):1235-1244. doi:10.1007/s00439-012-1152-8

39. Chen X, Wang Y, Cheng K, et al. Genetic variants in the regulatory region of SLC10A1 are not associated with the risk of hepatitis B virus infection and clearance. Infect Genet Evol. 2016;44:495-500. doi:10.1016/j.meegid.2016.07.043

40. Chang J, Zhong R, Tian J, et al. Exome-wide analyses identify lowfrequency variant in CYP26B1 and additional coding variants associated with esophageal squamous cell carcinoma. Nat Genet. 2018;50 (3):338-343. doi:10.1038/s41588-018-0045-8 
41. Schubbert S, Shannon K, Bollag G. Hyperactive Ras in developmental disorders and cancer. Nat Rev Cancer. 2007;7(4):295-308. doi:10. $1038 /$ nrc2109

42. Shaw RJ, Cantley LC. Ras, PI(3)K and mTOR signalling controls tumour cell growth. Nature. 2006;441(7092):424-430. doi:10.1038/ nature 04869

43. Okumura T, Ericksen RE, Takaishi S, et al. K-ras mutation targeted to gastric tissue progenitor cells results in chronic inflammation, an altered microenvironment, and progression to intraepithelial neoplasia. Cancer Res. 2010;70(21):8435-8445. doi:10.1158/0008-5472. CAN-10-1506

44. Benvenuti S, Sartore-Bianchi A, Di Nicolantonio F, et al. Oncogenic activation of the RAS/RAF signaling pathway impairs the response of metastatic colorectal cancers to anti-epidermal growth factor receptor antibody therapies. Cancer Res. 2007;67(6):2643-2648. doi:10.1158/0008-5472.CAN-06-4158

45. Ryan BM, Robles AI, Harris CC. Genetic variation in microRNA networks: the implications for cancer research. Nat Rev Cancer. 2010;10(6):389-402. doi:10.1038/nrc2867

46. Richardson K, Lai CQ, Parnell LD, Lee YC, Ordovas JM. A genomewide survey for SNPs altering microRNA seed sites identifies functional candidates in GWAS. BMC Genomics. 2011;12:504. doi:10.1186/1471-2164-12-504

47. Dai Q, Wei HL, Huang J, Zhou TJ, Chai L, Yang ZH. KRAS polymorphisms are associated with survival of CRC in Chinese population. Tumour Biol. 2016;37(4):4727-4734. doi:10.1007/s132 77-015-4314-1

48. Wang LE, Ma H, Hale KS, et al. Roles of genetic variants in the PI3K and RAS/RAF pathways in susceptibility to endometrial cancer and clinical outcomes. J Cancer Res Clin Oncol. 2012;138(3):377-385. doi:10.1007/s00432-011-1103-0
49. Crowley EH, Arena S, Lamba S, Di Nicolantonio F, Bardelli A. Targeted knock-in of the polymorphism rs61764370 does not affect KRAS expression but reduces let-7 levels. Hum Mutat. 2014;35 (2):208-214. doi:10.1002/humu.22487

50. Smits KM, Paranjape T, Nallur S, et al. A let-7 microRNA SNP in the KRAS 3'UTR is prognostic in early-stage colorectal cancer. Clin Cancer Res. 2011;17(24):7723-7731. doi:10.1158/1078-0432.CCR-11-0990

51. Zhang W, Winder T, Ning Y, et al. A let-7 microRNA-binding site polymorphism in 3'-untranslated region of KRAS gene predicts response in wild-type KRAS patients with metastatic colorectal cancer treated with cetuximab monotherapy. Ann Oncol. 2011;22 (1):104-109. doi:10.1093/annonc/mdq315

52. Graziano F, Canestrari E, Loupakis F, et al. Genetic modulation of the Let-7 microRNA binding to KRAS 3'-untranslated region and survival of metastatic colorectal cancer patients treated with salvage cetuximab-irinotecan. Pharmacogenomics J. 2010;10(5):458-464. doi:10.1038/tpj.2010.9

53. Gutierrez-Malacatt H, Ayala-Sanchez M, Aquino-Ortega X, et al. The rs61764370 functional variant in the KRAS oncogene is associated with chronic myeloid leukemia risk in women. Asian Pac J Cancer Prev. 2016;17(4):2265-2270. doi:10.7314/apjcp.2016.17.4.2265

54. Pharoah PD, Palmieri RT, Ramus SJ, et al. The role of KRAS rs61764370 in invasive epithelial ovarian cancer: implications for clinical testing. Clin Cancer Res. 2011;17(11):3742-3750. doi:10.1158/1078-0432.CCR-10-3405

55. Quaye L, Song H, Ramus SJ, et al. Tagging single-nucleotide polymorphisms in candidate oncogenes and susceptibility to ovarian cancer. Br J Cancer. 2009;100(6):993-1001. doi:10.1038/sj.bjc.660 4947

56. Tonini GP. Neuroblastoma by chance. J Cancer. 2019;10(12):26012603. doi:10.7150/jca.33291
OncoTargets and Therapy

\section{Publish your work in this journal}

OncoTargets and Therapy is an international, peer-reviewed, open access journal focusing on the pathological basis of all cancers, potential targets for therapy and treatment protocols employed to improve the management of cancer patients. The journal also focuses on the impact of management programs and new therapeutic

\section{Dovepress}

agents and protocols on patient perspectives such as quality of life, adherence and satisfaction. The manuscript management system is completely online and includes a very quick and fair peer-review system, which is all easy to use. Visit http://www.dovepress.com/ testimonials.php to read real quotes from published authors. 